\title{
Health-related restrictions of choices and choosing: implications for quality of life and clinical interventions
}

This article was published in the following Dove Press journal:

Patient Related Outcome Measures

7 August 2010

Number of times this article has been viewed

\author{
Barry J Gurland' \\ Huai Cheng ${ }^{2}$ \\ Mathew S Maurer ${ }^{3}$ \\ 'Columbia University Department of \\ Psychiatry, Stroud Center for Study \\ of Quality of Life, New York, NY, USA; \\ ${ }^{2}$ Anderson Cancer Center, Houston, \\ TX, USA; ${ }^{3}$ Columbia Presbyterian \\ Medical Center, New York, NY, USA
}

Background: The process of "accessing choices and choosing among them" (c-c) has been proposed as a model for understanding, evaluating, and assisting a patient's management of quality of life. If desired choices are freely accessible, and the act of choosing is efficient and unconstrained, then the outcome is optimized quality of life. The c-c model fits many clinical situations where improved quality of life is a goal, and interventions may be aimed at relieving health-related restrictions of the patient's desired activities.

Aims: To determine the impact of health restrictions of choices and choosing on indicators and outcomes reflecting quality of life.

Method: Secondary analysis of a community-based health survey of three ethnic groups, 65 years and older $(n=2,130)$, repeated after 18 months, with mortality over 6 years.

Findings: Complaints of health restrictions of desired activities accounted for about half the variance of all determinants of a quality of life proxy indicator, and had a high frequency. Such complaints also predicted declines in mood and function, higher death rates, and increased service use.

Conclusions: Clinical trials are warranted of the efficacy for quality of life improvement of interventions that focus on the relief of health-induced restrictions of desired activities.

Keywords: choice, quality of life, aging, health restrictions

\section{Introduction}

Providing choices to patients has become a central tenet of good quality clinical care. ${ }^{1-5}$ However, evaluating the outcomes of that care has been confounded by conflicting definitions of quality of life states and an overabundance of competing measures. ${ }^{6-9}$ Moreover, measures of quality of life are generally employed to evaluate interventions for health and related social conditions, not as guides to the selection or the development of that intervention. Similarly, the science base invoked in quality of life interventions has concentrated on measurement technology, or on the nature of the health disorders associated with impaired quality of life, rather than the nature of quality of life itself. Thus, there is a disconnect between the emphasis placed on the attention to quality of life severity and response to treatment, and the relatively little attention paid to how quality of life can impact the selection of the type of clinical intervention. For the same reasons, the science and evidence base for interventions aimed at improving a patient's quality of life are usually directed at the implicated health and associated disorders, not at the possible mechanisms relating to the nature of quality of life itself. We have argued that this relative neglect of specific causal pathways, which predispose health and related disorders to undermine the patient's
Correspondence: Barry J Gurland

Columbia University Department of

Psychiatry, Stroud Center for Study

of Quality of Life, 100 Haven Ave,

Tower 3 - Suite 30F, New York,

NY 10032, USA

$\mathrm{Tel}+|21278| 0600$

Email bjgl@columbia.edu 
quality of life, has hampered the search for more tailored and effective clinical interventions.

To address these conceptual, methodological, and clinical concerns, we previously presented ${ }^{10-12}$ a quality of life evaluation focusing on a process rather than a series of domain states. This single unifying adaptive process is held to manage the pursuit of desired well-being through accessing choices and choosing among them (the c-c model). The c-c model circumvents the dilemma inherent in judging the goodness or badness of states of quality in the life of another person when that judgment is "intrinsically ambiguous, defying useful certitude". ${ }^{10}$ We conjectured that consideration of the c-c process is necessary and sufficient for understanding health-related impairments of quality of life, and for evaluating and assisting persons when this process is constrained or inefficient. Restrictions or distortions of the c-c process are deemed to be rational targets for clinical interventions. If interventions free up access to desired choices, and reduce errors of fact and judgment in the act of choosing, then the outcome is expected to be improved quality of life. A person, when released from certain health-related restrictions, is then in a position to participate, with clinical help and guidance, in the relief of their distress.

The appeal of the c-c model of quality of life is that it (i) is already widely recognizable in health and long-term care service policy and practice, (ii) offers a unifying indicator of quality of life that avoids the conflicts and confounds in the current multiple alternative measures, (iii) leads to rational, client-specific, clinical interventions (elaborated later in the discussion section), and (iv) can be linked to scientific or other evidentiary spheres in a manner that keeps alive the prospect of progressively refining investigative and interventional approaches. For the latter purpose ("linking and refining" aim), we gave illustrative details in our previous paper $^{11}$ about evidence from evolutionary theory on how decision making plays a vital part in the pursuit of well-being and adaptation to the environment, from neuroscience insights into the mechanisms of attaching values to choices and reacting to those values, from cognitive psychology descriptions of the positive and negative influences on the efficiency of human decision making; from behavioral psychology on the personality typing of choices and choosing styles (eg, how people differ in their tolerance for increasing numbers of choices, in their manner of setting standards of satisfaction for the outcomes of choosing, and in the ability to adjust strategies of choosing in the light of experience). We also extracted relevant findings from comparative psychology on, for example, trade-off between the time taken in choosing and the outcomes of well-being, and observations from sociology, patho-physiology, and clinical investigations that extend the c-c framework into the relief of health- or context-imposed impairments of quality of life. Additionally, we constructed and described provisional guidelines for clinical approaches to relief of restrictions of the c-c process and restoration of its efficiency, where quality of life is compromised by health and related social problems.

The current paper examines empirical data on older persons for certain expectations raised by the c-c model: namely, that complaints of impediments to choices or choosing will (i) account for a substantial portion of variance in conventional indicators of quality of life, and (ii) will be substantially determined by specific, treatable health problems.

\section{Aims and hypotheses Aim}

To examine the c-c process as a determinant of conventional indicators of impairment of quality of life (depressed mood, unhappiness, and life dissatisfaction).

\section{Hypothesis}

Health-related restrictions of choices and choosing will account for a substantial portion of the variance in conventional indicators of quality of life.

\section{Methods}

\section{Study design and population}

The description of the study design and population has been previously published. ${ }^{13-15}$ Data were obtained from the North Manhattan Aging Project (NMAP), funded by the National Institute of Health at Columbia University. A random sample of Medicare beneficiaries were recruited, who were 65 years of age and older, and lived in a geographic area of 13 adjacent census tracts north of 150th Street in Manhattan (a multiethnic community). According to the 1990 census, 9,349 people older than 65 years lived in this area. The Health Care Financing Administration (HCFA) provided access to a random sample of approximately half of these recipients. In this group, 4,865 individuals, divided into 37 identical replicates representing the demographic characteristics of the cohort, were sent a letter from HCFA explaining that they had been randomly selected to participate in a study of aging. Subjects were drawn for interview by random methods, in replicated sub-samples, equally from the three cultural groups, until the numbers of elders in any group were exhausted, or the total of elders interviewed exceeded 2,100. Subsequently, it was determined that $470(9.7 \%)$ had died, $896(18.4 \%)$ no longer lived in the region, 47 (1\%) were ineligible, and 1,324 (37\%) did not wish 
to participate. The proportions of individuals within each ethnic group, as identified from HCFA records, differed only slightly between the total sample and those who participated (Total sample: African American, 35.4\%; Hispanic, 35.4\%; non-Hispanic White, 29.2\%. Participants: African American, 35.2\%; Hispanic, 38.9\%; non-Hispanic White, 25.8\%) Poverty, crime, and unemployment rates are high in the study target area. A total of 2,130 subjects were evaluated at baseline in 1993, and then, if consenting, revisited by trained interviewers at 18 month intervals over three years. The National Death Index was examined over a period of 6 years.

\section{Assessment technique}

Raters used a standard questionnaire, the Comprehensive Assessment and Referral Evaluation (CARE) ${ }^{16}$ with defined and coded responses, observations, and test protocols. Trained raters administered the interview in either English or Spanish, assisted by a computer program. (The CARE was first published in 1977). ${ }^{16}$

\section{Demographic and socioeconomic variables}

Gender; age; marital status; self-attributed ethno-racial group (Hispanic descent and, if not, non-Hispanic White, or AfricanAmerican); education (years of schooling: 0 to 4, 5 to 11, 12 or more); household income, as selected from a display card.

\section{Health variables}

A wide range of health problems was covered relevant to conventional quality of life, with particular attention to functioning and symptom syndromes in physical, cognitive, affective, social. and psychological domains, and patterns of health care utilization. ${ }^{17}$ The scales were previously derived using latent class analysis and have satisfactory content, clinical, and face validities, and inter-rater and internal consistency reliabilities, ${ }^{18-20}$ as well as concurrent and predictive validity for morbidity and mortality. Healthcare utilization included self-reported hospitalization, emergency room visits, and homecare.

\section{Definitions}

\section{Explicit complaints of restricted choices} and choosing

Explicit complaints of restricted c-c were elicited by eighteen questions about a health problem preventing the attainment of some desired goal (Felt Restrictions Scale). Either the health problem was specified and the impacted activity was left unspecified, or vice versa.

\section{Normative restrictions of choices and choosing}

Thirteen items covered engagement in social, occupational, and leisure activities. The normal frequency distribution in this older population is known (Social Engagement Scale).

\section{Implicit restrictions of choices and choosing:}

This definition embodies the view that choices and choosing is likely to be restricted in the presence of certain health problems, regardless of whether the subject makes that connection explicit; for examples, functional impairment, distressing symptom syndromes, or cognitive deficits.

\section{Statistical analysis}

The analyses presented are on baseline and first follow-up data from the NMAP study. For constructing the Felt Restrictions scale, the 18 constituent items were scored as 0 or 1 for absence or presence of the complaint, and in some instances as a third rating, 2, for degree of severity. A health services perspective was preserved by selecting only items that correlated significantly with a four-point rating of self-perceived severity of ill health. A Felt Restrictions scale score was obtained by adding the item values. Cronbach's alpha for reliability and internal consistency was used for evaluating the resulting scale.

Simple linear regressions (bivariate analysis) with one outcome variable (depressed mood and Felt Restriction, separately) and one independent variable (the individual health variables) were used to select independent variables at the level of $P<0.1$ for final multivariate linear regression analysis. Variables considered of special relevance (eg, race, income, and educational level) were automatically included in the final multivariate linear regression, even if bivariate analyses were not significant. Multivariate linear regression modeling was used to obtain adjusted associations of the two dependent variables (depressed mood as a continuum of severity on a 22 -item scale, and the Felt Restrictions scale, as described above) by several orders of entering. All analyses were performed in SPSS 17.0 (SPSS Inc, Chicago, IL). The influence of Felt Restrictions on outcomes at 18 months of depressed mood, function (mobility), and mortality were explored by employing Chi-square analyses.

\section{Human rights}

The Columbia University Institutional Review Board reviewed and approved this project. All individuals provided written informed consent. 


\section{Results}

\section{Demographics of the population}

The mean age was 74 and the range from 65 to 104 years, with about $31.2 \%$ of participants being greater than 80 years of age. The majority ( $46.8 \%$ and $33.7 \%$ ) were respectively Latino or African-American, with Whites being the minority (19.5\%). Females were $69.2 \%$, and educational levels were low, with $23.2 \%$ having less than 5 years of school, and only $32.1 \%$ with 12 or more years. Most (57.7\%) did not live alone, but only $28.5 \%$ were currently married. Income was severely limited. Annual household income was 10,506 dollars or less in $69.5 \%$ of the sample.

\section{Felt Restrictions scale}

The items used to identify Felt Restrictions are shown in Table 1, along with their prevalence in the population. Eleven out of the 18 Felt Restrictions scale items had a frequency over $20 \%$, and for 8 , it was $30 \%$ or more.

There is a fairly wide distribution of Felt Restrictions scale scores, with $23.9 \%$ of subjects scoring zero and another $14.8 \%$ reporting one restriction. $27.8 \%$ of subjects reported between 2 and 5 restrictions, $15.0 \%$ between 6 and 9 , and $18.5 \%$ of the population scored higher than the median of 9. (These proportions exclude $11.6 \%$ of the sample whose data were incomplete because of disorders affecting communication.) There are higher rates of felt restrictions after 75 years of age, and in females, lower income groups, non-married persons, and less-educated groups (all at $P<0.1$ ). Race is not associated with felt restrictions at the $P<0.1$ level.

\section{Reliability}

Cronbach's alpha index of reliability for the Felt Restrictions scale is 0.898 , showing high internal consistency. There was no evidence of redundancy between items, almost all item inter-correlations being below 0.60 .

\section{Bivariate associations of the dependent variables}

Using simple linear regression, the following health and relevant demographic variables were significant at the $P<0.1$ level with each of the two dependent variables (depressed mood and Felt Restrictions).

\section{Demographic associations}

Age, Gender, Not married, Race, Education, Monthly household income.

\section{Health, function, and well-being associations}

Basic ADL, IADL, Blocks without rest, Cognitive score, Illness incidents, Stroke, Respiratory syndrome, Cardiovascular syndrome, Pain, Fatigue, Social engagement, Isolation.

Notably, the correlation between Felt Restrictions and three prominent conventional indicators of quality of life, namely depressed mood, life dissatisfaction, and unhappiness,

Table I Felt Restrictions items and their frequencies

\begin{tabular}{ll}
\hline Items in Felt Restrictions scale & Item frequency \\
\hline Specific health cause of restrictions specified but restricted activity not specified & 46.4 \\
Not have enough energy to do the things wants to do & 35.4 \\
Changed way of doing things in order to reduce the chance of falling & 30.0 \\
Health gets in the way of doing the things wants to do & 23.9 \\
Difficulty with walking or getting around interferes with wants to do & 23.5 \\
Pain stops ordinary activities & 16.3 \\
Breathless, heart pounding, or exertion pain interferes with wants to do & 16.1 \\
Problems with hearing or seeing interfere with what wants to do & 12.6 \\
Problem with memory make it difficult to do accustomed things & \\
Specific health cause of restrictions not specified but restricted activity specified & 53.0 \\
Health stops from doing as much as would like the following: & 43.8 \\
Carrying heavy packages & 43.5 \\
Heavy chores & 31.7 \\
Paying job & 28.4 \\
Leisure & 25.2 \\
Getting around outside & 19.8 \\
Travel outside the neighborhood & 19.6 \\
Social activities & 7.5 \\
Light chores & 7.4 \\
Something else & \\
\hline
\end{tabular}


are $0.586,0.351$, and 0.211 respectively. This spread is not very different from the correlations between these indicators themselves: depressed mood and life dissatisfaction, 0.368 ; unhappiness and life dissatisfaction, 0.325; and depressed mood and unhappiness, 0.228 . Finally, using linear regression, self-perceived ill health is more strongly related to Felt Restrictions $(\mathrm{R} 2=0.246, \mathrm{r}=0.496)$ than is depressed mood, $(\mathrm{R} 2=0.174, \mathrm{r}=0.417)$, unhappiness $(\mathrm{R} 2=0.112, \mathrm{r}=0.336)$, or life dissatisfaction $(\mathrm{R} 2=0.179, \mathrm{r}=0.424)$.

\section{Regression on depressed mood}

For the regression analysis, we selected the 13 variables that were associated with depressed mood at the $P<0.01$ level of significance in bivariate analysis. The regression on depressed mood provided the following adjusted $\mathrm{R}$ values (Table 2). Entering Felt Restrictions first, R2 $=0.348$, after adding 13 control variables, $\mathrm{R} 2=0.593$ (change of 0.245 ), and with the demographic variables included, $\mathrm{R} 2=0.616$. This suggests that more then half of the association with depression of all health, social, and demographic variables could be explained or mediated by Felt Restrictions.

\section{Regression on unhappiness and life dissatisfaction}

Repeating the linear regression with the same control variables, with unhappiness (4 graded levels in a single item)

Table 2 Regression on depressed mood

\begin{tabular}{|c|c|}
\hline & $\begin{array}{l}\text { Depressed mood } \\
(0-22) \text { linear } \\
\text { regression, } \\
\text { adjusted } R 2\end{array}$ \\
\hline \multicolumn{2}{|l|}{ First order of entry } \\
\hline Felt Restrictions (I-I8) & 0.348 \\
\hline Second order of entry & 0.347 \\
\hline \multicolumn{2}{|l|}{ Normative restrictions: } \\
\hline \multicolumn{2}{|l|}{ Social engagement $(0-13)$} \\
\hline Third order of entry & 0.593 \\
\hline \multicolumn{2}{|l|}{ Implicit restrictions } \\
\hline \multicolumn{2}{|l|}{ Basic ADL (0-6); IADL (0-24) } \\
\hline \multicolumn{2}{|l|}{ Blocks without rest $(0,1-5,6)$; Cognitive score } \\
\hline \multicolumn{2}{|l|}{ (0-15); Illness incidents (0-9); Stroke (yes or no); } \\
\hline \multicolumn{2}{|l|}{ Respiratory syndrome (0-II); Cardiovascular } \\
\hline \multicolumn{2}{|l|}{ syndrome $(0-10)$; Pain $(0-14)$; Fatigue $(0-3,4+)$; } \\
\hline \multicolumn{2}{|l|}{ Isolation (0-6) } \\
\hline \multicolumn{2}{|l|}{ Demographics } \\
\hline $\begin{array}{l}\text { Age (65-74, 75-84, 85+); Gender (female); not } \\
\text { married; Race (White, Latino, African-American; }\end{array}$ & 0.616 \\
\hline \multicolumn{2}{|l|}{ Education (0-8 yr, some high school, high school } \\
\hline \multicolumn{2}{|l|}{ graduate, college and above); Income (monthly } \\
\hline \multicolumn{2}{|l|}{ income) } \\
\hline Total R2 & 0.616 \\
\hline
\end{tabular}

Notes: The number of items in each scale is given in parentheses. as the dependent variable, produced adjusted $\mathrm{R} 2$ values of 0.086, 0.093, 0.192, and 0.203 after the first, second, third, and entry of demographic indicators. With life dissatisfaction (3 graded levels in a single item) as the dependent variable, the corresponding adjusted R2 values were $0.112,0.129$, 0.151 , and 0.154 .

\section{Regression on Felt Restrictions}

For the Felt Restrictions regression (Table 3), we selected the 12 variables that were associated with Felt Restrictions at the $P<0.1$ level of significance in bivariate analysis. Entering Normative Restrictions first, R2 $=0.221$. After adding Implicit Restrictions (separate entry of eleven scales, each with multiple items) as control variables, $\mathrm{R} 2=0.737$ (change of 0.516). After adding demographic controls, the R2 increased only slightly (to 0.744 ). The major determinant of Implicit Restrictions is Explicit Restrictions. Normative restrictions had a substantial impact on Explicit Restrictions, but demographic influence was minimal.

\section{Longitudinal analyses}

Among those who reported Felt Restrictions at Time 1 (baseline) and were interviewed at follow-up 18 months after baseline $(n=1,419)$, the numbers who had the problem at follow-up (persistent) or no longer had the problem at follow-up (recovered) were examined for each item in the scale. The ratio of persistence over recovery, excluding one very low outlier, varied from a high of 3.23 to a low of 0.86 , with the majority (10 out of 18 ) being between 1.25 and 2.00. Odds ratio for prediction based on Felt Restrictions at baseline and deteriorated status at 18 months for function (limitation of blocks walked without rest) is 6.8 (5.2-8.9), for depressed mood, it is 2.8 (2.2-3.6), and for death rates over 6 years, it is $2.2(1.8-2.8)$.

The proportion of elders who used emergency room services during the previous year was $29.2 \%$ for those with Felt Restrictions (2+ score), versus $11.3 \%$ for those without Felt Restrictions (0-1 score). The corresponding proportions for an overnight stay in hospital during the year were $23.4 \%$ versus $7.0 \%$, and for use of homecare were $26.7 \%$ versus $2.2 \%$ (all Spearman $P<0.1$ ).

\section{Discussion}

The findings of this study are consistent with the stated hypotheses. Self-reported complaints ("felt restrictions"), which explicitly blame a health problem for restricting choices and choosing of desired activities, are associated with impairment of a conventional indicator (depressed mood) of 
Table 3 Regression on felt (explicit) restrictions

\begin{tabular}{|c|c|}
\hline & $\begin{array}{l}\text { FR }(0-18) \\
\text { linear regression, } \\
\text { adjusted } \mathbf{R} 2\end{array}$ \\
\hline First order of entry & 0.221 \\
\hline \multicolumn{2}{|l|}{ Normative restrictions } \\
\hline \multicolumn{2}{|l|}{ Social engagement $(0-13)$} \\
\hline Second order of entry & 0.737 \\
\hline \multicolumn{2}{|l|}{ Implicit restrictions } \\
\hline \multicolumn{2}{|l|}{ Basic ADL (0-6); IADL (0-24) } \\
\hline \multicolumn{2}{|l|}{ Blocks without rest $(0,1-5,6)$; Cognitive score } \\
\hline \multicolumn{2}{|l|}{ (0-I5); Illness incidents (0-9); Stroke (yes or no); } \\
\hline \multicolumn{2}{|l|}{ Respiratory syndrome (0-II); Cardiovascular } \\
\hline \multicolumn{2}{|l|}{ syndrome (0-I0); Pain (0-I4); Fatigue $(0-3,4+)$; } \\
\hline \multicolumn{2}{|l|}{ Isolation (0-6) } \\
\hline \multicolumn{2}{|l|}{ Demographics } \\
\hline \multicolumn{2}{|l|}{ married; Race (White, Latino, African-American; } \\
\hline \multicolumn{2}{|l|}{ Education (0-8 yr, some high school, high school } \\
\hline \\
\hline \multicolumn{2}{|l|}{ income) } \\
\hline Total R2 & 0.744 \\
\hline
\end{tabular}

Notes: The number of items in each scale is given in parentheses.

quality of life. A further range of health problems that appear at face value to be likely to restrict choices and choosing of desired activities, but are not spontaneously blamed for the restriction, are labeled implicitly (probably) restricting. We expect that a more penetrating probing of implicit restrictions would, in the great majority of instances, yield explicit complaints of restriction. That explicit and implicit restrictions occupy conceptually-related space is suggested by the high proportion of variance of the former (explicit) that is accounted for by the latter (implicit).

The explicit category, with only 18 items, was the strongest determinant of variance in depressed mood, even though the implicit category contained over one hundred items. It appears that elders can validly distinguish their health problems that are most likely, from those that are less likely, to restrict choices and choosing of desired activities. Clinically, this suggests that attention to explicitly felt restrictions should have priority in improving quality of life, and justifies the entry of explicit before implicit restrictions into the depressed mood regression. Nevertheless, implicit restrictions are also important targets for improving quality of life. The regression on impaired quality of life (depressed mood) suggests that if both explicit and implicit impediments are effectively relieved, then around $70 \%$ of impaired quality of life (as reflected in depressed mood) might be improved.

The high frequency of explicit restrictions in this elderly population (eg, $30 \%$ of the sample admitted that "health gets in the way of doing the things I want to do"), taken together with the accompanying increase in service demands, tendency to chronicity, and the worsening of the outcomes for declines in mood, function, and survival, indicates the size of the challenge to public health and clinical services, while the complexity of the challenge is underlined by the statistically significant, mutually adjusted associations between explicit restrictions and multiple health problems. These findings point to the need for targeted interventions in clinical, public health, and long-term care settings.

While the population studied was from a large community-dwelling population of older adults, they are also representative of older adult patients in health care settings, in that a high proportion (84.7\%) who explicitly complain of two or more health-imposed restrictions, were on one or more medications prescribed by a doctor (mainly for hypertension, heart disorder, or joint and muscle pain). Furthermore, among this group, $29.2 \%$ had made at least one visit to the emergency room during the year, $23.4 \%$ had been in hospital, and $26.7 \%$ were receiving home care.

Interventions can be designed to remove health and related restrictions on the patient's choices and choosing, thus strengthening their pursuit of quality of life. Details of the relevant clinical strategies and their derivation from a science base were covered, with citations, in a preceding paper, ${ }^{12}$ and are summarized here. The strategic elements are:

\section{Facilitating the c-c process}

It is essential to allow adequate time for the older patient to sift through available choices, with clear understanding of risks and benefits, and without distractions and pressures. Explicitly engaging the patient in the c-c process could deepen their involvement and counter the human tendency to rush to sub-optimal decisions. Also, by encouraging mid-course corrections of choosing in the light of experience of impacts, patients can realize the flexibility in this process.

\section{Personalizing the c-c process}

Assessing motives and intensity of participation in activities that are accessible to the patient. Identifying experiences previously enjoyed but no longer maintained, and the reasons for such restriction. Accommodating customary styles of choosing, such as tolerance for numbers of choices, search for optimal or sufficient satisfaction, and level of comfort with self-initiatives or deference to authority. 


\section{Relieving health imposed inhibitions and distortions of c-c}

The literature would suggest that especially relevant conditions include depression, obsessions, phobias, cognitive deficits, and many functional and symptomatic disorders such as chronic pain, fatigue, effort intolerance, mobility limitations, paralyses, and the like.

\section{Reinforcing positive expectations and responses}

Engagement in the choosing process can itself promote wellbeing independent of any removal of a formal restriction. Leaving a margin of uncertainty in expectations can improve outcomes.

\section{Structuring information gathering}

The clinical evaluation and outcome monitoring of interventions aimed at the c-c process can be assisted by use of structured questionnaires, most of which are sufficiently straightforward to permit self-administration by patients. The Felt Restrictions scale described in this paper is one example of such questionnaires. It is generic to a wide range of health conditions, but there are comparable generic instruments (for example, see Hays and Moralez, 2001) ${ }^{21}$ or those keyed to specific disorders (eg, The Living with Heart Failure Questionnaire of Guyatt et al, 1993).22

This study has several limitations. The data set includes self-reports that raise the possibility of combined measurement and reporting error. However, we did not include depressed mood among the determinants of Felt Restrictions. Had we done so as the last order of entry, it would have made a negligible difference of less than 0.01 to the total adjusted R2. For the sake of stimulating discussion with a wide readership, depressed mood was selected as a conventional indicator of quality of life, despite our having elsewhere ${ }^{10}$ expressed our reservations about the conceptual validity of such conventional indicators. Other conventional indicators of impaired quality of life, unhappiness, and life dissatisfaction were, as anticipated by the rationale for the c-c model, only modestly correlated with depressed mood, or with each other, or with felt restrictions.

Another limitation is that data were derived from a single, albeit large and multi-ethnic data set that was collected almost two decades ago, and was not designed specifically to examine the c-c model. The survey was set in a geographic area with generally low education, low income, and high crime rates. Accordingly, response rates were modest, and could have biased estimates of the frequency and associations of explicit and implicit restrictions. The substantial percentage of participants who were lost to follow-up limits our ability to evaluate outcomes.

Even if the associations presented here are consistent with a prospect of improving the quality of life outcomes for patients with health and related restrictions of desired activities, clinical trials would be necessary for demonstrating the efficacy of this paradigm. We contend that there is credible basis, and the outlines of a clinical method, for proceeding with such intervention trials.

\section{Disclosure}

The authors report no conflicts of interest in this work.

\section{References}

1. Dean M. Drive to improve patient choice in the UK NHS. Lancet. 2004;363(9405):302.

2. Emanuel EJ. Choice and representation in health care. Med Care Res Rev. 1999;56 (Suppl 1):113-140.

3. Meier DE, Morrison RS. Autonomy reconsidered. $N$ Engl J Med. 2002;346(14):1087-1089.

4. Morrison JL, Kirsch L, Mattingly PH, Rider ME, Dubow J. Consumerdirected health care plans: prospects for reshaping the organization, delivery, and responsibility of health care. Consumer Interests Annual. 2004;50.

5. Goldberg C. Bruce's Choice. Ann Intern Med. 2002;136(10):777-778.

6. Kahn RL, Juster FT. Well-Being: Concepts and Measures: J Soc Issues. 2002;58(4):627-644.

7. Hayry, M. Measuring the quality of life: why, how and what? In: Joyce CRB, O'Boyle CA, McGee H, editors. Individual quality of life: approaches to conceptualisation and assessment. Amsterdam: Harwood Academic Publishers; 1999:9-28.

8. Bullinger M. Assessing health related quality of life in medicine. An overview over concepts, methods and applications in international research. Restor Neurol Neurosci. 2002;20(3-4):93-101.

9. McKee M, Raine R. Choosing health? First choose your philosophy. Lancet. 2005;365(9457):369-371.

10. Gurland BJ, Gurland RV. The choices, choosing model of quality of life: description and rationale. Int J Geriatr Psychiatry. 2009;24(1):90-95.

11. Gurland BJ, Gurland RV. The choices, choosing model of quality of life: linkages to a science base. Int $J$ Geriatr Psychiatry. 2009;24(1):84-89.

12. Gurland BJ, Gurland RV, Toner J, Mitty E. Choices, choosing model of quality of life: clinical evaluation and intervention. J Interprof Care. 2009;23(2):110-120.

13. Cheng H, Maurer MS, Gurland B. Self-reported lack of energy (anergia) among elders in a multi-ethnic community. J Gerontol A Biol Sci Med Sci. 2008;63(7):707-714.

14. Gurland BJ, Wilder DE, Lantigua R, Stern Y, Chen J, Killeffer EH, Mayeux R. Rates of dementia in three ethnoracial groups. Int J Geriatr Psychiatry 1999;14(6):481-493.

15. Tang MX, Stern Y, Marder K, et al. The APOE-epsilon4 allele and the risk of Alzheimer disease among African Americans, whites, and Hispanics. JAMA. 1998;279(10):751-755.

16. Gurland B, Kuriansky J, Sharpe L, Simon R, Stiller P, Birkett P. The Comprehensive Assessment and Referral Evaluation (CARE) rationale, development and reliability. Int JAging Hum Dev 1977-1978; 8(1):9-42.

17. Gurland BJ, Wilder DE. The CARE interview revisited: development of an efficient, systematic clinical assessment. J Gerontol. 1984; 39(2):129-137. 
18. Teresi JA, Golden RR, Gurland BJ, Wilder DE, Bennett RG. Construct validity of indicator-scales developed from the Comprehensive Assessment and Referral Evaluation interview schedule. J Gerontol. 1984;39(2):147-157.

19. Golden RR, Teresi JA, Gurland BJ. Development of indicator scales for the Comprehensive Assessment and Referral Evaluation (CARE) interview schedule. J Gerontol. 1984;39(2):138-46.

20. Teresi JA, Golden RR, Gurland BJ. Concurrent and predictive validity of indicator scales developed for the Comprehensive Assessment and Referral Evaluation interview schedule. J Gerontol. 1984;39(2):158-165.
21. Hays RD, Morales LS. The RAND-36 measure of health-related quality of life. Ann Med. 2001;33(5):350-357.

22. Guyatt GH. Measurement of health-related quality of life in heart failure. J Am Coll Cardiol. 1993;22(4 Suppl A):185A-191A.

\section{Publish your work in this journal}

Patient Related Outcome Measures is an international, peer-reviewed, open access journal focusing on treatment outcomes specifically relevant to patients. All aspects of patient care are addressed within the journal and practitioners from all disciplines are invited to submit their work as well as healthcare researchers and patient support groups. Areas covered will include: Quality of life scores; Patient satisfaction audits; Treatment outcomes that focus on the patient; Research into improving patient outcomes; Hypotheses of interventions to improve outcomes; Short communications that illustrate improved outcomes; Case reports or series that show an improved patient experience; Patient journey descriptions or research.

\footnotetext{
Submit your manuscript here: http://www.dovepress.com/patient-related-outcome-measures-journal
} 